BMJ Open Diabetes Research \& Care

\title{
Insulin sensitivity predicts cognitive decline in individuals with prediabetes
}

Caroline Willmann, ${ }^{1,2,3}$ Kathrin Brockmann, ${ }^{4,5}$ Robert Wagner, ${ }^{1,2,3}$

Stephanie Kullmann, ${ }^{2,3}$ Hubert Preiss।, ${ }^{1,2,3,6,7}$ Günter Schnauder, ${ }^{1}$ Walter Maetzler, ${ }^{8}$ Thomas Gasser, ${ }^{4,5}$ Daniela Berg, ${ }^{4,8}$ Gerhard W Eschweiler, ${ }^{9,10}$ Florian Metzger, ${ }^{9,10,11}$ Andreas J Fallgatter, ${ }^{9,10}$ Hans-Ulrich Häring, ${ }^{1,2,3}$ Andreas Fritsche, ${ }^{1,2,3}$

Martin Heni (10) $1,2,3,12$

To cite: Willmann $\mathrm{C}$, Brockmann K, Wagner R, et al. Insulin sensitivity predicts cognitive decline in individuals with prediabetes. BMJ Open Diab Res Care 2020;8:e001741. doi:10.1136/ bmjdrc-2020-001741

- Supplemental material is published online only. To view please visit the journal online (http://dx.doi.org/10.1136/ bmjdrc-2020-001741).

Received 29 June 2020 Revised 13 0ctober 2020 Accepted 16 October 2020

\section{Check for updates}

(c) Author(s) (or their employer(s)) 2020. Re-use permitted under CC BY-NC. No commercial re-use. See rights and permissions. Published by BMJ.

For numbered affiliations see end of article.

Correspondence to Dr Martin Heni; martin.heni@med.unituebingen.de

\section{ABSTRACT}

Introduction Epidemiological studies indicate an association between type 2 diabetes and cognitive dysfunction that appear to start already in the prediabetic state. Although cross-sectional studies have linked insulin resistance to impaired cognition, the potential predictive value of insulin resistance has not yet been sufficiently studied longitudinally without confounding by overt diabetes (and its pharmacological treatment). Research design and methods We investigated longitudinal data from participants of the 'Tübinger Evaluation of Risk Factors for Early Detection of Neurodegeneration' Study. Subjects underwent a neurocognitive assessment battery (CERAD Plus battery; Consortium to Establish a Registry for Alzheimer's Disease) at baseline and followed every 2 years (median follow-up 4.0 Q1-3: 2.2-4.3 years). Subjects within a pre-diabetic glycated hemoglobin range of $5.6 \%-6.5 \%$ underwent 5 -point $75 \mathrm{~g}$ oral glucose tolerance tests (OGTTs) with assessment of insulin sensitivity and insulin secretion $(n=175)$. Subjects with newly diagnosed diabetes mellitus or with major depressivity (Beck Depression Inventory $>20)$ were excluded $(n=15)$. Data were analyzed by mixed models using sex, age and glycemic trait as fixed effects. Subject and time since first measurement were used as random effects.

Results Insulin sensitivity was positively associated with the CERAD sum score (higher is better) in a timedependent manner $(p=0.0057)$. This result is mainly driven by a steeper decrease in the memory domain associated with lower insulin sensitivity $(p=0.029)$. The interaction between age and insulin sensitivity was independent of glycemia $(p=0.02)$. There was also no association between insulin secretion and cognition.

Conclusions Insulin resistance rather than sole elevation of blood glucose predicts cognitive decline, specifically in the memory domain, in persons with prediabetes. Treatments of diabetes that improve insulin sensitivity might therefore have the potential to postpone or even prevent cognitive decline in patients with diabetes.

\section{INTRODUCTION}

The epidemiological association of type 2 diabetes mellitus with cognitive dysfunction ranging from mild cognitive impairment to manifest dementia is well established. ${ }^{1}$ This

\section{Significance of this study}

What is already known about this subject?

- Epidemiological studies indicate an association between type 2 diabetes and cognitive dysfunction that already starts in the prediabetic state.

What are the new findings?

> Insulin resistance rather than sole elevation of blood glucose predicts cognitive decline, specifically in the memory domain, in persons with prediabetes.

How might these results change the focus of research or clinical practice?

> Treatments of diabetes that improve insulin sensitivity might therefore have the potential to postpone or even prevent cognitive decline in patients with diabetes.

association already starts in midlife; and the longer the duration of diabetes, the higher the risk for developing slight cognitive impairment and even dementia. ${ }^{1}$ Moreover, cognitive decline seems to start well ahead of the manifestation of overt diabetes, in the prediabetic state. ${ }^{2}$ In order to understand the time line of progression and the interplay of these pathologic entities, studies in subjects at high risk for diabetes or dementia are needed.

The pathogenetic link between impaired glycemic control and cognitive decline has not yet been entirely elucidated. Reduced insulin secretion and peripheral insulin resistance are the major pathomechanisms in the development of type 2 diabetes mellitus. Several studies have linked peripheral insulin resistance in healthy subjects with impaired cognitive performance. However, only a minority of them had a longitudinal study design and they all assessed glucose metabolism from fasting glucose and/or fasting insulin levels only. ${ }^{3-9}$ These measurements cannot sufficiently address all major aspects of glucose metabolism, as humans are in the 
postprandial state most of the daytime. Therefore, standardized challenge tests provide more precise measurements of glucose metabolism and insulin sensitivity.

We therefore investigated whether traits of glucose metabolism including insulin sensitivity and insulin secretion are associated with future cognitive performance and might predict cognitive decline in non-diabetic subjects. As opposed to most previous studies, our study assessed traits of glucose metabolism by a standardized 5-point $75 \mathrm{~g}$ oral glucose tolerance test (OGTT) at one time point. This allowed us to obtain more precise information on glucose tolerance status, insulin sensitivity and insulin secretion. Comprehensive neurocognitive assessment was performed by the extended Consortium to Establish a Registry for Alzheimer's Disease (CERAD Plus) test battery at baseline and every second year after baseline assessment.

\section{RESEARCH DESIGN AND METHODS}

The subjects were recruited from the ongoing prospective longitudinal Tübinger Evaluation of Risk Factors for Early Detection of Neurodegeneration (TREND) Study, which conducts biennial assessments of elderly participants without neurodegenerative diseases but with at least one of the prodromal markers for neurodegeneration (depression, hyposmia or probable REM-sleep-behaviour disorder). Moreover, participants had no prior history of stroke, inflammatory disorders affecting the central nervous system (such as multiple sclerosis, encephalitis, meningitis, vasculitis) and inability to walk without aid. ${ }^{10}$ Of this ongoing TREND Study, 175 subjects with a prediabetic glycated hemoglobin (HbA1c) within a range of $5.6 \%$ and $6.5 \%$ underwent OGTT. In three subjects, diabetes was diagnosed from this OGTT according to the classification of the American Diabetes Association. ${ }^{11}$ These subjects were excluded from all further analysis. Furthermore, subjects with manifest depression (Beck Depression Inventory (BDI) scores $>20$ ) were also excluded from this analysis as depression represents a confounder for cognitive testing. This resulted in data from 160 participants that were finally analyzed for this study. Detailed subject characteristics at baseline are given in table 1. Subject characteristics and data of cognitive tests at different time points are shown in online supplemental table 1.

\section{Assessment of cognitive function and depression}

Cognition was tested using the established, standardized German version of the extended CERAD Plus neuropsychological battery. ${ }^{12}$ The battery contains the following subtests: semantic and phonematic verbal fluency tasks, Boston Naming Test, Mini Mental Status Examination, word list learn, word list recall, word list recognition, constructional praxis, recall of constructional praxis and the trail-making test parts $\mathrm{A}$ and $\mathrm{B}$. The subtests of the CERAD Plus battery were grouped into four domains: executive, memory, language and

\begin{tabular}{|c|c|}
\hline Gender (female / male) (n) & $64 / 96$ \\
\hline Age (years) & $64.6 \pm 0.43(44-82)$ \\
\hline $\mathrm{BMI}\left(\mathrm{kg} / \mathrm{m}^{2}\right)$ & $26.3 \pm 0.31(18-39)$ \\
\hline $\begin{array}{l}\text { Glucose tolerance status } \\
\text { (NGT/IFG/IFG+IGT/IGT) (n) }\end{array}$ & $92 / 36 / 14 / 18$ \\
\hline Fasting glucose (mM) & $5.38 \pm 0.04$ \\
\hline Glucose, 120 min OGTT (mM) & $6.40 \pm 0.12$ \\
\hline Fasting insulin (pM) & $75.24 \pm 4.27$ \\
\hline $\mathrm{HbA1c}(\%)$ & $5.84 \pm 0.02$ \\
\hline Insulin sensitivity Index, ISI (AU) & $10.99 \pm 0.46$ \\
\hline Insulinogenic Index, IGI (AU) & $158.69 \pm 8.64$ \\
\hline Disposition Index, DI (AU) & $2.58 \pm 0.18$ \\
\hline Habitual Physical Activity Score & $8.49 \pm 0.1$ \\
\hline Systolic blood pressure $(\mathrm{mm} \mathrm{Hg})$ & $140 \pm 1.5$ \\
\hline Diastolic blood pressure $(\mathrm{mm} \mathrm{Hg})$ & $87 \pm 0.9$ \\
\hline Total cholesterol (mg/dl) & $208 \pm 3$ \\
\hline HDL cholesterol (mg/dl) & $57 \pm 1$ \\
\hline LDL cholesterol (mg/dl) & $118 \pm 2$ \\
\hline BDI & $4.7 \pm 0.34$ \\
\hline Years of education (years) & $13.81 \pm 0.62$ \\
\hline Smokers/past smokers (n) & $11 / 49$ \\
\hline Known and treated hypertension (n) & 44 \\
\hline $\begin{array}{l}\text { Known and treated } \\
\text { hypercholesterinemia }(n)\end{array}$ & 29 \\
\hline
\end{tabular}

BDI, Beck Depression Inventory; BMI, body mass index; HbA1c, glycated hemoglobin; HDL, high-density lipoprotein; IFG, impaired fasting glucose; IGT, impaired glucose tolerance; ISI, Insulin Sensitivity Index; LDL, low-density lipoprotein; NGT, normal glucose tolerance; OGTT, oral glucose tolerance test.

visuospatial. This cognitive assessment battery was performed at baseline and every 2 years afterwards (median follow-up 4.0 years Q1-3: 2.2-4.3 years, online supplemental table 1). The CERAD Plus test battery sum score was calculated according to Chandler. ${ }^{13}$

Depressive symptoms were assessed with the BDI-I. ${ }^{14}$ The time line of the study is presented as online supplemental figure 1 .

\section{Oral glucose tolerance test}

Subjects with a HbA1c of $5.6 \%-6.5 \%$ without known diabetes were metabolically investigated by a 5 -point $75 \mathrm{~g}$ OGTT with assessment of insulin sensitivity and insulin secretion $(\mathrm{n}=175)$. After an overnight fast, subjects ingested a standard solution containing $75 \mathrm{~g}$ glucose (Accu-Chek Dextro O.G.T., Roche Diagnostics, Mannheim, Germany) at 08:00 hours. Subsequent glucose and insulin concentrations were determined from venous blood samples that had been obtained at $0,30,60,90$ and $120 \mathrm{~min}$. 


\section{Glucose metabolism}

Serum insulin was determined on an ADVIA Centaur XP immunoassay system (Siemens Healthineers, Eschborn, Germany). Blood glucose was measured by the oxidase method (EKF Diagnostic, Barleben, Germany). Insulinogenic Index (IGI) was calculated as (Insulin 30-Glucose $0)$. The OGTT-derived Insulin Sensitivity Index (ISI) was calculated as ISI $=10000 \sqrt{ }($ Glucose $0 \cdot$ Insulin $0 \cdot$ Glucose mean.Insulin mean), as proposed by Matsuda and DeFronzo. ${ }^{15}$ The Disposition Index (DI) was calculated as ISI $\times$ IGI to account for the individual degree of insulin resistance. As an alternative to the Matsuda Index, we also computed the non-esterified fatty acid (NEFA)-ISI Index from insulin and free fatty acid levels during OGTT. This has been suggested to be a more robust marker of insulin sensitivity in comparison to gold-standard methods measuring insulin sensitivity. ${ }^{16}$

\section{Statistical analysis}

All analyses were performed with R V.3.4. The association of glycemic traits (insulin sensitivity and insulin secretion) with the change of cognitive scores was examined using linear mixed models with random slopes and random intercepts. All available results from repeated cognitive testing were included into the analyses. Prior to modeling, glycemic traits were log-transformed and cognitive scores were inverse-normal transformed to obtain normally distributed variables. Outliers above or below more than 5 SD from the mean were excluded (out of 10843 data values that were available for the reported analyses, 126 values were excluded from analyses). The linear mixed models were fitted with the lme4 package using sex, age at each measurement and glycemic trait as fixed effects. Subject and elapsed time since first measurement were applied as random effects. The following model formula was used: neurologic_outcome sex + age * metabolic_variable $+(1+$ time $\mid$ subject_id $)$. The null hypothesis was no interaction between age at each measurement and glycemic variable, which would suggest that the glycemic status does not modulate the age-dependent slope (corresponding to the natural change) of the cognitive score. In order to reduce multicollinearity and enable detection of marginal effects (age on cognitive score, glycemic trait on cognitive score), age and glycemic trait were standardized (scaled to a mean of 0 with an SD of 1 , for glycemic traits this step was performed after log-transformation). Variables were Z-score transformed (centered and scaled) for fitting mixed models and for improvement of the interpretation of model coefficients. ${ }^{17}$

We also modeled the risk of a cognitive decline $>5 \%$ for the cognitive score using Kaplan-Meier estimators. Here, we performed a median cut for insulin sensitivity, which led to a comparison of a stratum with high and low insulin sensitivities. The two survival curves of the median-splitted insulin sensitivity groups were compared using log-rank tests. Unless otherwise stated, values are reported as mean \pm SEM.

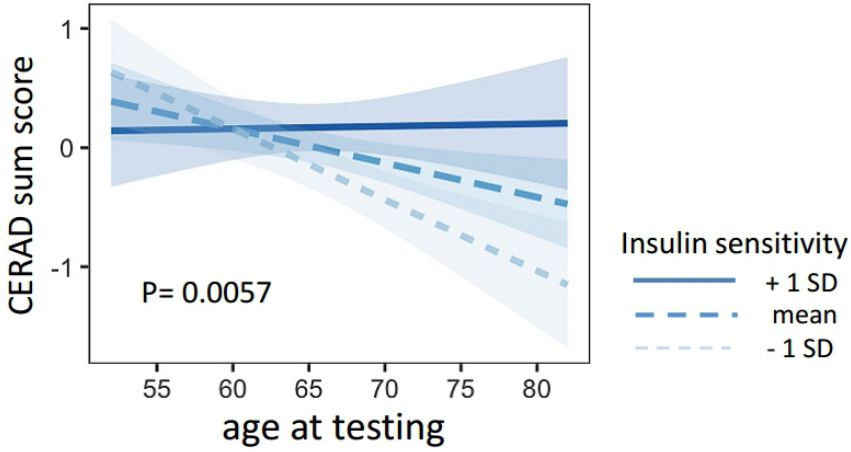

Figure 1 Presented are the regression lines from the statistical models. The linear trend lines show the association of age and the CERAD sum score for different levels of insulin sensitivity (mean $\pm 1 \mathrm{SD}$ ). CERAD, Consortium to Establish a Registry for Alzheimer's Disease.

\section{RESULTS}

As assessed during the OGTT, 92 subjects were normal glucose tolerant (NGT), 36 had impaired fasting glucose (IFG), 18 had impaired glucose tolerance (IGT) and 14 had both IGT and IFG. Detailed subject characteristics at baseline are given in table 1 . The three subjects newly diagnosed with diabetes during the OGTT were excluded from further analysis. Data sets of 160 participants at baseline, of 149 participants after 2 years and 78 participants after 4 years could be analyzed.

The CERAD sum score decreased (ie, worsened) with age $(\beta=-0.890 .26, p=0.0009)$ and was positively associated with insulin sensitivity assessed by the Matsuda Index $(\beta=0.330 .13, p=0.015)$.

Insulin sensitivity was associated with the timedependent change of CERAD sum score (interaction for age $\times$ insulin sensitivity, $\beta=0.320 .11, p=0.0057)$. Lower insulin sensitivity was associated with a steeper decline of the CERAD sum score (figure 1). This interaction was also replicated with an alternative ISI, the NEFA-ISI Index $(\beta=0.360 .13, p=0.0083)$. The interaction between age $\times$ insulin sensitivity (Matsuda Index) on CERAD sum score furthermore remained significant after adjustment for BMI, systolic and diastolic blood pressure, triglycerides and cholesterol $(\beta=0.170 .06, \mathrm{p}=0.0057)$.

When analyzing the different cognitive domains (memory, executive, language and visuospatial domains), there was an interaction between insulin sensitivity and age on the memory domain performance $(\beta=0.27 \pm 0.12$, $\mathrm{p}=0.029$ ), while no interactions on the other cognitive domains were detected (all $\mathrm{p}>0.05$, table 2 ).

Insulin secretion (assessed by the DI), was neither associated with the CERAD sum score nor with the tested subdomains (all p>0.16, table 2).

Similarly to the CERAD sum score, tests assessing memory function worsened with age (word list recall $\beta=-0.860 .24, p=0.00052$, word list learn $\beta=-0.61 \pm 0.26$, $\mathrm{p}=0.022$ ) and there was a trend towards a positive association with insulin sensitivity (word list recall $\beta=-0.23 \pm 0.0 .12$, $\mathrm{p}=0.063$; word list learn $\beta=0.24 \pm 0.13, \mathrm{p}=0.067$ ) (table 3). 
Table 2 Interaction of age at cognitive tests and insulin sensitivity and insulin secretion on the different cognitive domains

\begin{tabular}{lllllll}
\hline Cognitive test & Glycemic trait & $\boldsymbol{\beta}$ & $\mathbf{E S E M}$ & $\mathbf{P}$ & $\begin{array}{l}\mathbf{t}- \\
\text { statistic }\end{array}$ & $\begin{array}{l}\text { Degrees of } \\
\text { freedom }\end{array}$ \\
\hline CERAD sum score & Insulin sensitivity & 0.32 & 0.11 & $\mathbf{0 . 0 0 5 7}$ & 2.8 & 257 \\
& Insulin secretion & 0.094 & 0.093 & 0.31 & 1 & 255 \\
Memory domain & Insulin sensitivity & 0.27 & 0.12 & $\mathbf{0 . 0 2 9}$ & 2.2 & 202 \\
& Insulin secretion & 0.14 & 0.097 & 0.16 & 0.63 & 170 \\
Executive domain & Insulin sensitivity & 0.13 & 0.12 & 0.3 & 1 & 226 \\
& Insulin secretion & 0.019 & 0.096 & 0.84 & 0.2 & 224 \\
Language domain & Insulin sensitivity & 0.096 & 0.12 & 0.43 & 0.79 & 269 \\
& Insulin secretion & 0.048 & 0.096 & 0.62 & 0.5 & 264 \\
Visuospatial domain & Insulin sensitivity & 0.16 & 0.11 & 0.17 & -0.33 & 196 \\
& Insulin secretion & -0.029 & 0.09 & 0.74 & 1.4 & 198 \\
\hline
\end{tabular}

$p$ values below 0.05 are given in bold.

CERAD, Consortium to Establish a Registry for Alzheimer's Disease.

There was a steeper decline over time in insulin-resistant subjects (word list recall $\beta=0.31 \pm 0.11, \mathrm{p}=0.0036$; world list learn $\beta=0.24 \pm 0.11, p=0.037$ ) (figure 2 , table 3 ).

All above listed interactions remained significant after adjusting for area under the curve (AUC) ${ }_{0-120}$ glucose, that is, glycemia during the OGTT (all $\mathrm{p} \leq 0.04$ ). Moreover, all listed interactions remained significant after adjusting for BMI (all $\mathrm{p} \leq 0.029$ ). The interaction between insulin sensitivity and age on the CERAD sum score also remained significant after adjusting for cardiovascular risk factors that could potentially influence cognition (hypertension, hypercholesterinemia and smoking, $\mathrm{p}=0.01$ ).

Besides the OGTT-derived Matsuda ISI, we also analyzed fasting insulin resistance (homeostatic model assessment of insulin resistance (HOMA-IR)). Comparable results were obtained, however, effect sizes were higher for the Matsuda Index (online supplemental table 2).

When modeling the risk of a decline $>5 \%$ for the cognitive score using Kaplan-Meier estimators, the more insulin-resistant half of the subjects had a significantly higher risk for cognitive decline assessed by CERAD sum score (figure 3). The 95\% CIs for the proportional hazards (Cox) model were -1.56 to $-0.2, \mathrm{p}=0.01$ with estimates $-0.88 \pm 0.35$.

\section{CONCLUSIONS}

In the present study, we detected insulin resistance as a significant modulator of cognitive decline in persons at risk for type 2 diabetes. In contrast, we did not find associations of insulin secretion with the tested cognitive variables. These results support and extend previous studies linking peripheral insulin resistance to cognitive decline. ${ }^{3-9}$ Of note, the association was independent of glycemia, suggesting that not elevated blood sugar per se but rather insulin resistance could be an important factor in the underlying mechanism that drives cognitive decline.

Insulin signaling in the brain has been shown to play a pivotal role in cognition which is summarized in a recent review of our group. ${ }^{18}$ There is a strong correlation between peripheral insulin sensitivity and central insulin action, ${ }^{19-22}$ at least for some brain areas centrally involved in memory and executive function. ${ }^{18}$ Thus, our findings of impaired cognitive function in insulin-resistant

Table 3 Interaction of insulin sensitivity and age on cognitive memory scores 'word list recall' and 'word list learn'

\begin{tabular}{llrlllc}
\hline & & & & & \multicolumn{2}{c}{$\begin{array}{c}\text { Degrees } \\
\text { of }\end{array}$} \\
& & $\boldsymbol{\beta}$ & \pm SEM & P value & \multicolumn{2}{c}{ t-statistic freedom } \\
\hline word list recall & Age & -0.86 & 0.24 & $\mathbf{0 . 0 0 0 5 2}$ & -3.5 & 269 \\
& Age $\times$ insulin sensitivity & 0.31 & 0.11 & $\mathbf{0 . 0 0 3 6}$ & 2.9 & 279 \\
& Insulin sensitivity & 0.23 & 0.12 & $\mathbf{0 . 0 6 3}$ & 1.9 & 135 \\
word list learn & Age & -0.61 & 0.26 & $\mathbf{0 . 0 2 2}$ & -2.3 & 242 \\
& Age $\times$ insulin sensitivity & 0.24 & 0.11 & $\mathbf{0 . 0 3 7}$ & 2.1 & 244 \\
& Insulin sensitivity & 0.24 & 0.13 & $\mathbf{0 . 0 6 7}$ & 1.8 & 140 \\
\hline
\end{tabular}

$p$ values below 0.05 are given in bold. 

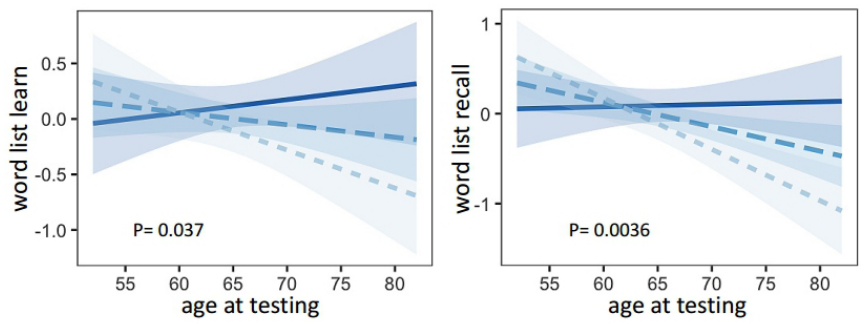

Insulin sensitivity $+1 S D$ $-1 \mathrm{SD}$

Figure 2 Presented are the regression lines from the statistical models. The linear trend lines show the association of age and the cognitive memory tests 'world list learn' (left) and 'word list recall' (right) for different levels of insulin sensitivity (mean $\pm 1 \mathrm{SD})$.

subjects are likely due to altered insulin signaling in the brain. ${ }^{23}$ Insulin receptors are densely expressed in brain areas such as the hippocampus and prefrontal cortex that are involved in memory function. ${ }^{24}{ }^{25}$ Correspondingly, we found effects in regards to insulin sensitivity especially in the memory domain.

Another possible hypothesis is that inflammation might be the pathophysiological link between the two diseases. Chronic inflammation is often observed in insulin-resistant subjects and leads to tissue alteration not only in the periphery but also in the brain. ${ }^{18}$ As our current data cannot prove the mechanistic link between insulin sensitivity and cognition, further studies are still needed to elucidate this issue.

To our knowledge, there is only one longitudinal study investigating the link between glucose metabolism and overt dementia with precise metabolic phenotyping. ${ }^{26}$ The association of low insulin sensitivity with a higher risk of dementia did not withstand adjustments for other risk factors (including diabetes) in this study. However, its primary and secondary end points were manifest Alzheimer's disease (AD) or

\section{CERAD sum score}

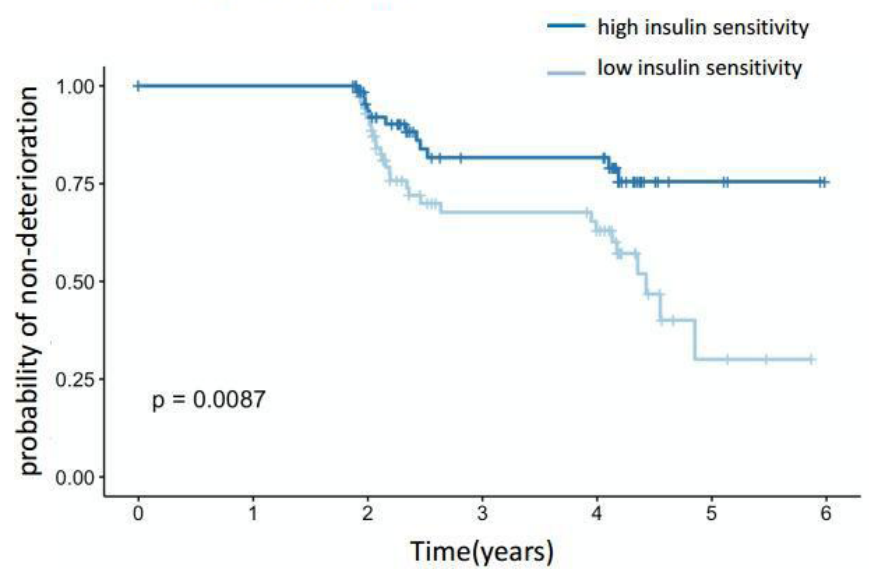

Figure 3 Risk of cognitive decline (assessed by CERAD sum score) $>5 \%$ using Kaplan-Meier estimators. Subjects were stratified by the median of insulin sensitivity. Insulinresistant subjects are represented in the lower curve, insulinsensitive subjects in the upper curve. CERAD, Consortium to Establish a Registry for Alzheimer's Disease. vascular dementia, and even though a subgroup was classified with 'any dementia or cognitive impairment', the study might have missed the association of insulin sensitivity with an only slight decline in cognitive performance that would be expected in otherwise healthy persons. The same study found insulin secretion to be associated with an increased risk of $\mathrm{AD}$ in a population with a mean age of 71 years at baseline,$^{26}$ a finding that we could not replicate in our younger subjects with a mean age of 64 years. The main differences are the longer duration of the other study with an older baseline age and a median follow-up of 12 years. Our current analysis had a median follow-up of 4 years but covered an age range from 44 years to 82 years (median 65 years), resulting in an observation period of 640 patient years. Of note, insulin secretion and insulin resistance are well correlated in subjects without diabetes. In our study, the use of a cohort in transition from normal glucose regulation to diabetes could have increased the sensitivity to precisely detect associations between metabolism and cognition. Although our data are observational, very recent results from large genetic studies demonstrate a genetic association between $\mathrm{AD}$ and diabetes, suggesting that the link might indeed be causal. ${ }^{27}$

One limitation of our current study is the relatively small sample size. However, we improved statistical power by using age at each measurement and glycemic trait as fixed effects and subject and elapsed time since first measurement as random effects. Furthermore, the number of patients with IFG and/or IGT was smaller than the number of persons with NGT. This proportion is, however, in line with previous findings ${ }^{28}{ }^{29}$ and underlines that HbAlc and OGTT detect different phenotypes of disturbed glucose metabolism. Also, we had no follow-up values of metabolic traits available for analyses. Such follow-ups will be of particular interest when following the progression from prediabetes towards overt diabetes, but would not affect the predictive value of insulin sensitivity at baseline.

Our current findings altogether suggest the need of an early intervention in persons at risk for diabetes and dementia that targets insulin resistance. Treatment strategies for diabetes that improve insulin sensitivity could have the potential to postpone cognitive decline in such patients. In fact, insulin-sensitizing peroxisome proliferator-activated receptor (PPAR) $\gamma$ agonists were shown to preserve or improve cognitive function in patients with $\mathrm{AD}$, in some, but not all studies. ${ }^{30}{ }^{31}$ Also, restoring insulin signaling in the brain, for example, by intranasal insulin administration led to improvement of cognitive memory processes in healthy persons as well as patients with $\mathrm{AD},{ }^{31}{ }^{32}$ again emphasizing the role of brain insulin signaling/sensitivity in cognitive function. One further approach for the treatment of cognitive impairments has recently gained growing interest glucagon-like peptide-1 (GLP-1) receptor agonists. ${ }^{3133}$ These drugs showed first beneficial effect in recent pilot trials ${ }^{34-36}$ and larger trials are ongoing. ${ }^{31} 33$ Of note, animal studies indicate a close interaction between GLP-1 receptor and insulin receptor signaling in the brain. ${ }^{33738}$ Thus, the cognitive benefits of GLP-1 receptor agonists could at least partly be modulated via improved brain insulin sensitivity. 
In summary, insulin resistance is associated with cognitive decline and specifically memory impairment in persons at increased risk for diabetes. Therefore, insulin resistance rather than sole elevation of blood glucose could predict cognitive impairment. Therapeutic interventions improving insulin sensitivity might therefore have the potential to postpone or even prevent cognitive decline.

\section{Author affiliations}

'Department of Internal Medicine IV, University Hospital of Tübingen, Tübingen, Germany

${ }^{2}$ Institute for Diabetes Research and Metabolic Diseases (IDM) of the Helmholtz Center Munich at the University of Tübingen, Helmholtz Center Munich, Tübingen, Germany

${ }^{3}$ German Centre for Diabetes Research (DZD), Neuherberg, Germany

${ }^{4}$ Department of Neurodegeneration and Hertie-Institute for Clinical Brain Research, Center of Neurology, University of Tübingen, Tübingen, Germany

${ }^{5}$ German Center for Neurodegenerative Diseases (DZNE), University of Tübingen, Tübingen, Germany

${ }^{6}$ Institute for Diabetes and Obesity, Helmholtz Diabetes Centre at Helmholtz Zentrum München, German Research Centre for Environmental Health (GmbH), Neuherberg, Germany

${ }^{7}$ Department of Pharmacy and Biochemistry, Institute of Pharmaceutical Sciences, University of Tübingen, Tübingen, Germany

${ }^{8}$ Department of Neurology, Christian-Albrechts University, Kiel, Germany

${ }^{9}$ Department of Psychiatry and Psychotherapy, University Hospital Tübingen, Tübingen, Germany

${ }^{10}$ Geriatric Center at the University Hospital of Tübingen, Tübingen, Germany

${ }^{11}$ Department of Psychiatry and Psychotherapy, Vitos Hospital Haina, Haina, Germany

${ }^{12}$ Department for Diagnostic Laboratory Medicine, Institute for Clinical Chemistry and Pathobiochemistry, University Hospital Tübingen, Tübingen, Germany

Acknowledgements The authors thank all the study participants for their cooperation with this project. The authors thank the clinical study teams at the University of Tübingen, Germany for technical assistance. The authors thank the Deutsche Forschungsgemeinschaft and the Open Access Publishing Fund of the University of Tübingen, Germany, for their support.

Contributors CW researched and analyzed data and drafted the manuscript, KB researched data and contributed to discussion, RW analyzed data and contributed to discussion, SK, HP contributed to discussion, GS contributed to discussion, WM, TG, DB contributed to the design of the study and to discussion, GWE, FM contributed to discussion, $\mathrm{H}-\mathrm{UH}, \mathrm{AF}, \mathrm{MH}$ contributed to the design of the study and to discussion. All authors approved the final version of the manuscript prior to submission.

Funding Part of this work was supported by a grant (01GI0925) from the Federal Ministry of Education and Research (BMBF) to the German Center for Diabetes Research (DZD e.V.). The TREND Study has been, and still is supported by the Hertie Institute for Clinical Brain Research, the German Centre for Neurodegenerative Diseases (DZNE within the framework of iMed Helmholtz Association), the Centre for Integrative Neuroscience, TEVA Pharmaceutical Industries Ltd., Union Chimique Belge (UCB), Janssen Pharmaceuticals, Inc. and the International Parkinson Fonds. The supporting institutions had no influence on the design, conduct, or analysis of the study.

Competing interests None declared.

Patient consent for publication Not required.

Ethics approval Informed written consent was obtained from study participants prior inclusion in the study and the ethics committee of the University of Tübingen approved the protocol (90/2009B02 and 350/2007B01).

Provenance and peer review Not commissioned; externally peer reviewed.

Data availability statement The data are not publicly available because they contain information that could compromise research participant privacy/consent.

Supplemental material This content has been supplied by the author(s). It has not been vetted by BMJ Publishing Group Limited (BMJ) and may not have been peer-reviewed. Any opinions or recommendations discussed are solely those of the author(s) and are not endorsed by BMJ. BMJ disclaims all liability and responsibility arising from any reliance placed on the content. Where the content includes any translated material, BMJ does not warrant the accuracy and reliability of the translations (including but not limited to local regulations, clinical guidelines, terminology, drug names and drug dosages), and is not responsible for any error and/or omissions arising from translation and adaptation or otherwise.

Open access This is an open access article distributed in accordance with the Creative Commons Attribution Non Commercial (CC BY-NC 4.0) license, which permits others to distribute, remix, adapt, build upon this work non-commercially, and license their derivative works on different terms, provided the original work is properly cited, appropriate credit is given, any changes made indicated, and the use is non-commercial. See: http://creativecommons.org/licenses/by-nc/4.0/.

ORCID iD

Martin Heni http://orcid.org/0000-0002-8462-3832

\section{REFERENCES}

1 Cheng G, Huang C, Deng H, et al. Diabetes as a risk factor for dementia and mild cognitive impairment: a meta-analysis of longitudinal studies. Intern Med J 2012;42:484-91.

2 Yaffe K, Blackwell T, Whitmer RA, et al. Glycosylated hemoglobin level and development of mild cognitive impairment or dementia in older women. J Nutr Health Aging 2006;10:293-5.

3 Ekblad LL, Rinne JO, Puukka P, et al. Insulin resistance predicts cognitive decline: an 11-year follow-up of a nationally representative adult population sample. Diabetes Care 2017;40:751-8.

4 Lutski M, Weinstein G, Goldbourt U, et al. Insulin Resistance and Future Cognitive Performance and Cognitive Decline in Elderly Patients with Cardiovascular Disease. J Alzheimers Dis 2017;57:633-43.

5 Young SE, Mainous AG, Carnemolla M. Hyperinsulinemia and cognitive decline in a middle-aged cohort. Diabetes Care 2006;29:2688-93.

6 Kilander L, Nyman H, Boberg M, et al. Hypertension is related to cognitive impairment: a 20-year follow-up of 999 men. Hypertension 1998;31:780-6.

7 van Oijen M, Okereke OI, Kang JH, et al. Fasting insulin levels and cognitive decline in older women without diabetes. Neuroepidemiology 2008;30:174-9.

8 Okereke OI, Kurth T, Pollak MN, et al. Fasting plasma insulin, C-peptide and cognitive change in older men without diabetes: results from the physicians' health study II. Neuroepidemiology 2010;34:200-7.

9 Euser SM, Sattar N, Witteman JCM, et al. A prospective analysis of elevated fasting glucose levels and cognitive function in older people: results from prosper and the Rotterdam study. Diabetes 2010:59:1601-7.

10 Hobert MA, Niebler R, Meyer SI, et al. Poor trail making test performance is directly associated with altered dual task prioritization in the elderly--baseline results from the TREND study. PLoS One 2011;6:e27831.

11 American Diabetes Association. (2) classification and diagnosis of diabetes. Diabetes Care 2015;38 Suppl:S8-16.

12 Morris JC, Mohs RC, Rogers H, et al. Consortium to establish a Registry for Alzheimer's disease (CERAD) clinical and neuropsychological assessment of Alzheimer's disease. Psychopharmacol Bull 1988;24:641-52.

13 Chandler MJ, Lacritz LH, Hynan LS, et al. A total score for the CERAD neuropsychological battery. Neurology 2005;65:102-6.

14 Beck AT, Ward CH, Mendelson M, et al. An inventory for measuring depression. Arch Gen Psychiatry 1961;4:561-71.

15 DeFronzo RA. Insulin resistance, lipotoxicity, type 2 diabetes and atherosclerosis: the missing links. The Claude Bernard lecture 2009. Diabetologia 2010;53:1270-87.

16 Wagner R, Fritsche L, Heni M, et al. A novel insulin sensitivity index particularly suitable to measure insulin sensitivity during gestation. Acta Diabetol 2016;53:1037-44

17 Gelman A. Scaling regression inputs by dividing by two standard deviations. Stat Med 2008;27:2865-73.

18 Kullmann S, Heni M, Hallschmid M, et al. Brain insulin resistance at the crossroads of metabolic and cognitive disorders in humans. Physiol Rev 2016;96:1169-209.

19 Heni M, Kullmann S, Preissl H, et al. Impaired insulin action in the human brain: causes and metabolic consequences. Nat Rev Endocrinol 2015;11:701-11.

20 Heni M, Wagner R, Kullmann S, et al. Hypothalamic and striata insulin action suppresses endogenous glucose production and may 
stimulate glucose uptake during hyperinsulinemia in lean but not in overweight men. Diabetes 2017;66:1797-806.

21 Heni M, Wagner R, Kullmann S, et al. Central insulin administration improves whole-body insulin sensitivity via hypothalamus and parasympathetic outputs in men. Diabetes 2014;63:4083-8.

22 Ruud J, Steculorum SM, Brüning JC. Neuronal control of peripheral insulin sensitivity and glucose metabolism. Nat Commun 2017;8:15259.

23 Butterfield DA, Halliwell B, stress O. Oxidative stress, dysfunctional glucose metabolism and Alzheimer disease. Nat Rev Neurosci 2019;20:148-60.

24 Havrankova J, Roth J, Brownstein M. Insulin receptors are widely distributed in the central nervous system of the rat. Nature 1978;272:827-9.

25 Kullmann S, Kleinridders A, Small DM, et al. Central nervous pathways of insulin action in the control of metabolism and food intake. Lancet Diabetes Endocrinol 2020;8:524-34.

26 Rönnemaa E, Zethelius B, Sundelöf J, et al. Glucose metabolism and the risk of Alzheimer's disease and dementia: a populationbased 12 year follow-up study in 71-year-old men. Diabetologia 2009;52:1504-10.

27 Jansen IE, Savage JE, Watanabe K, et al. Genome-Wide metaanalysis identifies new loci and functional pathways influencing Alzheimer's disease risk. Nat Genet 2019;51:404-13.

28 Peter A, Fritsche A, Stefan N, et al. Diagnostic value of hemoglobin A1c for type 2 diabetes mellitus in a population at risk. Exp Clin Endocrinol Diabetes 2011;119:234-7.
29 Ang SH, Thevarajah M, Alias $\mathrm{Y}$, et al. Current aspects in hemoglobin A1c detection: a review. Clin Chim Acta 2015;439:202-11.

30 Galimberti D, Scarpini E. Pioglitazone for the treatment of Alzheimer's disease. Expert Opin Investig Drugs 2017;26:97-101.

31 Kellar D, Craft S. Brain insulin resistance in Alzheimer's disease and related disorders: mechanisms and therapeutic approaches. Lancet Neurol 2020;19:758-66

32 Chapman CD, Schiöth HB, Grillo CA, et al. Intranasal insulin in Alzheimer's disease: food for thought. Neuropharmacology 2018;136:196-201.

33 Hölscher C. Evidence for pathophysiological commonalities between metabolic and neurodegenerative diseases. Int Rev Neurobiol 2020;155:65-89.

34 Vadini F, Simeone PG, Boccatonda A, et al. Liraglutide improves memory in obese patients with prediabetes or early type 2 diabetes: a randomized, controlled study. Int J Obes 2020;44:1254-63.

35 Gejl M, Gjedde A, Egefjord L, et al. In Alzheimer's disease, 6-month treatment with GLP-1 analog prevents decline of brain glucose metabolism: randomized, placebo-controlled, double-blind clinical trial. Front Aging Neurosci 2016;8:108.

36 Mullins RJ, Mustapic M, Chia CW, et al. A pilot study of exenatide actions in Alzheimer's disease. Curr Alzheimer Res 2019;16:741-52.

37 Sandoval D, Sisley SR. Brain GLP-1 and insulin sensitivity. Mol Cell Endocrinol 2015;418 Pt 1:27-32.

38 Batista AF, Forny-Germano L, Clarke JR, et al. The diabetes drug liraglutide reverses cognitive impairment in mice and attenuates insulin receptor and synaptic pathology in a non-human primate model of Alzheimer's disease. J Pathol 2018;245:85-100. 